\title{
Systematic characterization of a novel gal operon in Thermoanaerobacter tengcongensis
}

Correspondence
Siqi Liu
siqiliu@genomics.org.cn

Received 28 October 2008

Revised 15 January 2009

Accepted 16 January 2009
Zhong Qian, ${ }^{1,2}$ Bo Meng, ${ }^{1,2}$ Quanhui Wang, ${ }^{3}$ Zhuowei Wang, ${ }^{1,2}$ Chuanqi Zhou, ${ }^{1,2}$ Qian Wang, ${ }^{1,2}$ Shuyang Tu, ${ }^{1,2}$ Liang Lin, ${ }^{1,2}$ Yanhe $\mathrm{Ma}^{3}$ and Siqi Liu ${ }^{1,2}$

\author{
${ }^{1}$ Beijing Institute of Genomics, Chinese Academy of Sciences, Beijing 101318, PR China \\ ${ }^{2}$ Beijing Proteomics Institute, Beijing 101318, PR China \\ ${ }^{3}$ Institute of Microbiology, Chinese Academy of Sciences, Beijing 100101, PR China
}

\section{INTRODUCTION}

Many prokaryotes can utilize galactose as a carbon source. Several different uptake systems transport galactose into the cytoplasm, while a unique pathway, the Leloir pathway (Frey, 1996), subsequently converts the galactose to glucose 1-phosphate. The Leloir pathway is known to be composed of gene products from either the lac-gal regulon (Vaillancourt et al., 2002; Vaughan et al., 2001) or the gal operon (Ajdic \& Ferretti, 1998; Bettenbrock \& Alpert, 1998; Mackie \& Wilson, 1972; Rohde et al., 2000; Weickert \& Adhya, 1993). The lac-gal regulon consists of the genes involved in lactose and galactose metabolism, such as galRKTEM-lacSZ in Streptococcus thermophilus (Vaughan et al., 2001), while the gal operon contains the genes that participate in galactose metabolism, such as galETKM in

Abbreviations: EMSA, electrophoretic mobility shift assay; HTH, helixturn-helix; LC, liquid chromatography; 5' RACE, 5' rapid amplification of cDNA ends.

Two supplementary figures, showing gene expression of the gal operon at different culture temperatures and phylogenetic analysis of gal genes in $T$. tengcongensis alongside gal genes in other thermophiles, are available with the online version of this paper.
Escherichia coli. Up to now, the lac-gal regulon and the gal operon have been reported in Proteobacteria and Firmicutes. The molecular regulation of the gal operon in Proteobacteria such as E. coli is well known (Roy et al., 2004; Semsey et al., 2002, 2004, 2006), but the study of Firmicute gal operons is considered to be uncharted territory. Firmicutes are broadly divided into two classes, bacilli and clostridia. Five operons related to galactose metabolism have been characterized in lactic acid bacteria, such as Lactobacillus casei (Bettenbrock \& Alpert, 1998), Lactococcus lactis (Luesink et al., 1998), Streptococcus mutans (Ajdic \& Ferretti, 1998), Streptococcus salivarius (Vaillancourt et al., 2002) and S. thermophilus (Vaillancourt et al., 2002), including three gal-lac regulons and two gal operons. These operon structures are different from those of E. coli because of the proximity of the GalR of bacilli to other structural genes. In contrast, neither the gal operon nor the gal-lac regulon has ever been reported in clostridia.

Thermoanaerobacter tengcongensis belongs to the clostridia, and can survive temperatures from 50 to $80{ }^{\circ} \mathrm{C}$ (Xue et al., 2001). Its genomic sequence was determined in 2002 (Bao 
et al., 2002), and the proteome at the optimal temperature has also been determined (Wang et al., 2004). T. tengcongensis can utilize several carbon sources, such as glucose, galactose and lactose. Investigations of galactose metabolism in $T$. tengcongensis have revealed the lack of a lac-gal regulon, but genomic data suggest the existence of a putative gal operon. The putative gal operon is composed of five genes, TTE1929, TTE1928, TTE1927, TTE1926 and TTE1925. In addition, the functional domains of these genes have been analysed. TTE1929, TTE1928 and TTE1927 are annotated as GalT, GalK and GalE, respectively, TTE1926 as GalR of the ROK (repressor, open reading frame and kinase) family (Titgemeyer et al., 1994), and TTE1925 as a hypothetical protein. On the other hand, the regulator of this putative operon, GalR, is predicted to be not, as usual, divergently transcribed (Ajdic \& Ferretti, 1998; Mackie \& Wilson, 1972; Rohde et al., 2000), but in the same direction as the other four genes. Since this was the first investigation of the gal operon in clostridia, it was desirable that the theoretical model could be supported by experimental data. Hence, the regulation of the $T$. tengcongensis gal operon and the functions of its genes were investigated.

\section{METHODS}

Culture of $\boldsymbol{T}$. tengcongensis. T. tengcongensis strain $\mathrm{MB}^{4 \mathrm{~T}}\left({ }^{\mathrm{T}}\right.$, type strain) was cultured in modified complex MB medium (Xue et al., 2001), using mannitol, galactose or glucose as carbon source, and tryptone as nitrogen source. The bacteria were routinely cultured at $75{ }^{\circ} \mathrm{C}$ if not otherwise mentioned. The cells were harvested, washed twice with PBS and centrifuged at $3000 \mathrm{~g}$ for $5 \mathrm{~min}$ at $4{ }^{\circ} \mathrm{C}$, and the pellets were collected for preparation of RNA and protein.

Total RNA extraction from $\boldsymbol{T}$. tengcongensis. Total RNA from T. tengcongensis harvested at exponential phase was extracted by TRIzol reagent (Invitrogen). The concentration of RNA was photometrically estimated at $A_{260}$ by an SMG3000 spectrophotometer (BGI). The quality of RNA was examined by conventional agarose electrophoresis.

RT-PCR analysis of intergenic regions of the gal operon. RTPCR was done with M-MLV reverse transcriptase (Promega) according to the manufacturer's instructions. Total RNA templates for RT-PCR were treated with DNase (Takara) multiple times until no DNA product was detected by Taq DNA polymerase in a PCR with any of the RT-PCR primers listed in Supplementary Table S1.

Real-time PCR analysis of gal genes in T. tengcongensis. cDNAs were generated through reverse transcription catalysed by MMLV reverse transcriptase (Promega) with total RNA as the template. The gene amplification was carried out in a PCR machine (Eppendorf) with designed primers (Supplementary Table S1). To quantitatively estimate gal gene expression, real-time PCR was carried out in a PRISM 7300 real-time PCR system (ABI). cDNA libraries of T. tengcongensis generated from differently cultured bacteria were used as the templates for real-time PCR, and the lengths of PCR products were $\sim 200 \mathrm{bp}$. The $\mathrm{Ct}$ values for each PCR were normalized against the Ct of $16 \mathrm{~S}$ rRNA. Data were calculated according to relative gene expression by the $2^{-\Delta \Delta C t}$ formula (Li et al., 2006). In addition, melting curves for each PCR were analysed carefully to ensure the purity of the amplification products. $5^{\prime}$ Rapid amplification of cDNA ends (5' RACE) analysis of gal operon promoters. Total RNA of $T$. tengcongensis cultured in galactose medium was extracted as described above. The transcription starting sites of $P_{\text {gal } 1}$ and $P_{\text {gal } 2}$ were determined with a SMART RACE cDNA Amplification kit (Clontech) according to the user manual. SubPCR (second PCR reaction) was carried out with specific primers and with the RACE products as templates. The subPCR products were purified with an AxyPrep DNA Gel Extraction Kit (Axygen) and sequenced (BGI).

Expression of recombinant gal proteins and generation of corresponding antibodies. The gal genes were overexpressed in $E$. coli BL21 (DE3). Recombinant proteins were purified through an $\mathrm{Ni}^{2+}$ affinity column (Qiagen) and used to generate antibodies. The rabbit sera were collected and purified by protein A affinity chromatography (Bio-Rad). Titres and specificities of the antibodies were routinely examined by ELISA and Western blotting.

Protein identification by Western blot analysis. Equal amounts of protein lysate $(15 \mu \mathrm{g})$ of each sample, quantified by the Bradford method, were separated on $12 \%$ SDS-PAGE and transferred onto PVDF membranes (Millipore) for Western blot analysis (Wang et al., 2007). Each Western blot was run in parallel at least three times.

To monitor protein degradation in vitro, each recombinant gal protein at the same quantity was incubated for $1 \mathrm{~h}$ with the lysate of $T$. tengcongensis cultured in galactose medium at 75 or $80{ }^{\circ} \mathrm{C}$, and was detected by Western blotting using anti-His-tag antibody (Cell Signalling).

Electrophoresis mobility shift assay (EMSA) and EMSA-liquid chromatography (LC) MS-MS. Based on bioinformatic analysis, probes were designed for the regions upstream of the operon and potentially interacting with regulatory elements, as follows: $5^{\prime}$ TTTCTTTTGAAGACTTAATTAAAAAATATAATAAAGTCTGTGAGGGAAAGAAAATGACTCAAAAAG , termed $O_{1}$; and $5^{\prime}$-TAAACCTTTCACATAAAACAAAATTTGTTGAGGGTATATTGTAGTGCATTAGAAGT, termed $\mathrm{O}_{2}$ (potential protein binding sites shown in bold type). Also, another two probes, overlapping $\mathrm{O}_{1}$ and $\mathrm{O}_{2}$, were designed: $O_{1}{ }^{\prime}, 5^{\prime}$-TATTGTAACTGCTATTGTTGACATACCAGGCGCTTTTCTTTTGAAGACTTAATTAAA; and $\mathrm{O}_{2}{ }^{\prime}, 5^{\prime}$-CGAATATAATAAAAAAGATGTTAATTAAAATAATAAATAAACCTTTCACATAAAACAAA (overlapping regions underlined). These single-strand DNA probes were synthesized, denatured, labelled with $\left[\gamma^{32} \mathrm{P}\right] \mathrm{ATP}$ by T4 polynucleotide kinase (NEB), and mixed with different concentrations of lysate of galactose-cultured T. tengcongensis, or recombinant TTE1926 or TTE1925 protein (as the negative control) for EMSA (Wu et al., 2007).

The DNA-protein complexes in T. tengcongensis were analysed by EMSA-LC MS-MS according to Stenger's method, with modifications (Stenger et al., 2004). DNA-protein complexes 1A and 2A, which refer, respectively, to probe $O_{1}$ and $O_{2}$ incubated with lysate of galactose-cultured $T$. tengcongensis, were isolated by EMSA. The shifted bands were then excised and digested with trypsin (Promega). Finally, the peptides were separated by HPLC (UltiMate 3000, Dionex) and identified by MS (HCT Ultra, Bruker). For analysis of LC MS-MS data, stringent criteria were adopted: (1) all the identified proteins should be acquired from duplicate preparations and injections; (2) all the identified proteins should be elicited from more than three matched peptides; (3) all the identified proteins should co-appear under both sets of experimental conditions $-O_{1}$ or $\mathrm{O}_{2}$ alone would be considered non-specific.

Enzyme assays. Mutarotase assays were carried out according to the modified method of Majumdar et al. (2004) using a P-1030 polarimeter (Jasco). The rate of nonenzymic turnover was subtracted from the initial rate of the enzymic reaction. The first-order rate 
constant was obtained from plots of the values of angular rotation against reaction time:

$K=\ln \left[\left(\alpha_{0}-\alpha_{\mathrm{e}}\right) /\left(\alpha_{\mathrm{t}}-\alpha_{\mathrm{e}}\right)\right] \times 1 / t$

where $K$ is the rate constant, $\alpha_{0}, \alpha_{\mathrm{t}}$ and $\alpha_{\mathrm{e}}$ the observed angular rotation, and $t$ the reaction time (Brahma \& Bhattacharyya, 2004).

Galactokinase (GalK) activity was measured by the NADH-ADP coupling assay (Mustapha et al., 1995; Qian et al., 2006). The kinetic parameters were calculated with SigmaPlot 8.0 (SPSS). The activities of GalT and GalE were assayed as described by Lai et al. (1999) and Maxwell et al. (1962), and one unit was defined as the change in $A_{340}$ in $1 \mathrm{~min}$ in the presence of $1 \mu \mathrm{g}$ protein.

\section{RESULTS}

\section{Bioinformatic analysis of the putative gal operon in T. tengcongensis}

Based upon the genomic data, a putative gal operon in the region from 1539 to $7763 \mathrm{bp}$ in section 170 of 244 sections of the complete genome was deduced. As shown in Fig. 1(a), TTE1929 was annotated as galactose-1-phosphate uridylyltransferase (galT), catalysing the formation of glucose 1phosphate and UDP-galactose from UDP-glucose and galactose 1-phosphate. TTE1928 was annotated as galactokinase $(\mathrm{galK})$, catalysing the formation of galactose 1phosphate from galactose. TTE1927 was annotated as UDP-glucose 4-epimerase ( $\mathrm{galE}$ ), catalysing the interconversion of UDP-glucose and UDP-galactose. Analysis of the TTE1929 upstream sequence by Neural Network Promoter Prediction (http://www.fruitfly.org/seq_tools/promoter. html) revealed one putative promoter and two putative operators. At the -10 region of the predicted promoter, the sequence TATAAT was a typical Pribnow box. The sequence TTGACG was predicted to be the -35 region (Fig. 1b). Downstream of TTE1925, a stem-loop structure with a calculated free energy $\left(\Delta G^{0}\right)$ of $-10.01 \mathrm{kcal} \mathrm{mol}^{-1}(-41.9 \mathrm{~kJ}$ $\mathrm{mol}^{-1}$ ) (data not shown) was located as a potential transcriptional terminator. TTE1926 was noted to be a member of the ROK family, with a helix-turn-helix (HTH) structure in the $\mathrm{N}$ terminus, the typical DNA-binding motif in regulators such as GalR. Moreover, transcription of GalR in most reported gal operons is regulated by its own promoter and the direction of transcription is divergent to that of the other gal genes (Vaillancourt et al., 2002; Weickert \& Adhya, 1993), but TTE1926 is located within the gal operon, and is co-transcribed with the other gal genes in the same direction and without its own promoter. Such a novel structure of the gal operon has, to our knowledge, never been reported in thermophilic bacteria. Through sequence analysis, no conserved domain was detected for TTE1925. Hence, exploring the function of TTE1925 required biochemical experiments. Taking all the bioinformatic data together, it is proposed that $T$. tengcongensis possesses a novel type of gal operon.

\section{Gene expression of the T. tengcongensis gal operon induced by galactose}

If the genes were located within an operon, they would be co-transcribed. Thus, four pairs of PCR primers (2928, (a)

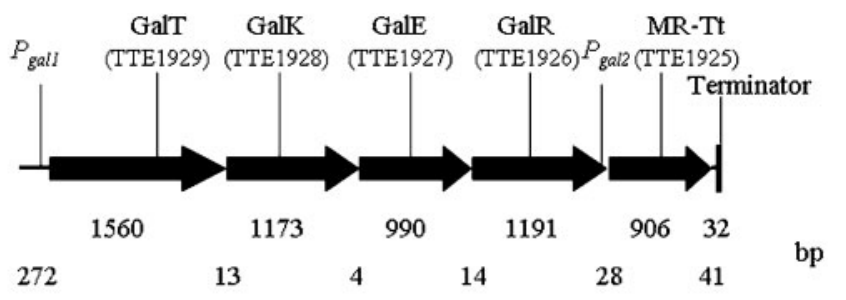

(b)

$P_{\text {gall }}$ :

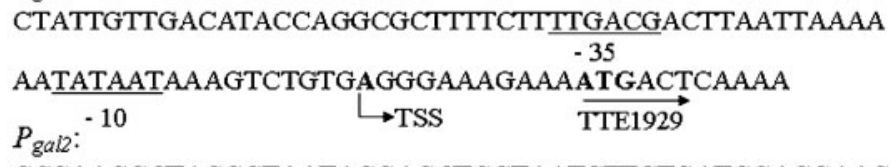

CCCAACGCTACGCTAATAGGAGCTGCTAATCTTGTGATGGACGAAG
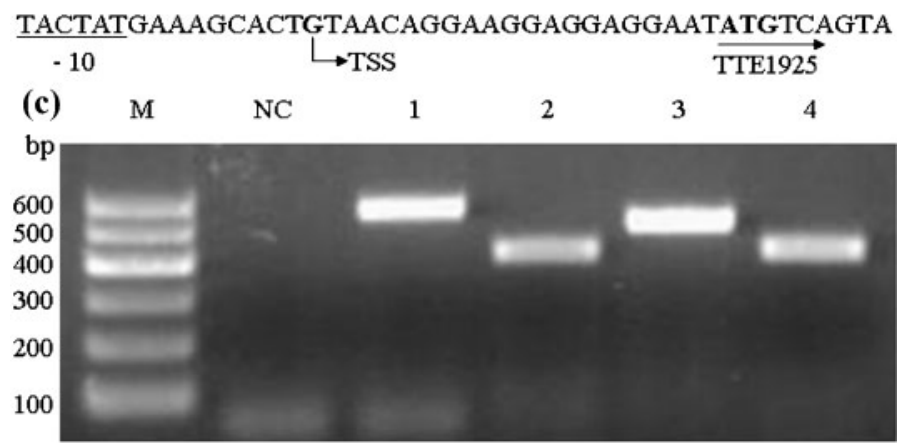

Fig. 1. Components of the $T$. tengcongensis $\mathrm{gal}$ operon, as confirmed by intergenic analysis with RT-PCR. (a) Gene organization of the gal operon in T. tengcongensis. Arrows indicate the direction of gene transcription. The lengths (in bp) of genes (upper numbers) and intergenic regions (lower numbers) are given. (b) Nucleotide sequences of $P_{\text {gal } 1}$ and $P_{\text {gal2 }}$. TSS, transcriptional start site. (c) Agarose gel electrophoresis of RT-PCR amplification products of the intergenic regions of the $g$ al operon. Lanes: M, DNA ladder; NC, negative control reaction performed without reverse transcriptase to test for RNA purity; 1, 2, 3 and 4, amplified intergenic regions of TTE1929TTE1928, TTE1928-TTE1927, TTE1927TTE1926 and TTE1926-TTE1925, respectively. 
2827, 2726 and 2625; Supplementary Table S1) were designed to amplify the intergenic regions between neighbouring gal genes from $T$. tengcongensis cDNA. Also, a negative control was designed to eliminate the possibility of genomic DNA contamination (lane NC in Fig. 1c). As shown in Fig. 1(c), four predicted fragments spanning the intergenic regions were detected, suggesting that all the given pairs were linked in a transcript.

As a gal operon, its transcription as well as translation should respond to galactose induction. The expression of the gal operon genes in T. tengcongensis cultured in glucose or galactose medium was measured at the mRNA and the protein level for comparison of the inducing response. Analysed by real-time PCR, the relative mRNA abundance of gal genes acquired from galactose-cultured T. tengcongensis was significantly higher than that from glucosecultured cells (Fig. 2a). Specifically, the fold-increase in mRNA due to galactose induction was 5.6 for TTE1925, 4.5 for TTE1926, 4.5 for TTE1927, 9.2 for TTE1928 and 2.3 for TTE1929. Using Western blotting, the relative protein abundance of the gal proteins from galactose-cultured $T$. tengcongensis was also significantly higher than that from glucose-cultured cells (Fig. 2b). Specifically, the fold increases of the protein were 2.9 for TTE1925, infinite for TTE1926, 14.3 for TTE1927, 10.3 for TTE1928 and 13.7 for TTE1929. To confirm that the gene expression of the gal operon specifically responded to galactose induction, $T$. tengcongensis also was cultured in $\mathrm{MB}$ medium containing mannitol, a neutral carbon source. No gal gene expression except that of TTE1925 was found. The expression of $g a l$ proteins was specifically activated by the presence of galactose in the medium (Fig. 3a). These results thus reinforced the argument that gal gene expression is only induced by galactose and not by other carbon sources. Interestingly, there was a relatively high background of TTE1925 in the glucose-cultured $T$. tengcongensis. This prompted the question of whether there was a promoter located at upstream of TTE1925.

The dynamic profile of protein expression was then monitored. When $T$. tengcongensis prior-cultured in glucose was transferred to galactose medium, the expression of gal proteins responded to the stimulus within a short period. After $2 \mathrm{~h}$ galactose induction, the expression of gal proteins was greatly increased from an undetectable to a detectable level. After $8 \mathrm{~h}$, the protein content reached a maximum steady state (Fig. 3b, c). As mentioned above, although TTE1925 expression was significantly induced by galactose, it remained at a detectable level in $T$. tengcongensis cultured in glucose medium (Fig. 2b). Moreover, if T. tengcongensis prior-cultured in galactose was transferred to glucose medium, the relative protein content of gal proteins was significantly decreased after $4 \mathrm{~h}$,
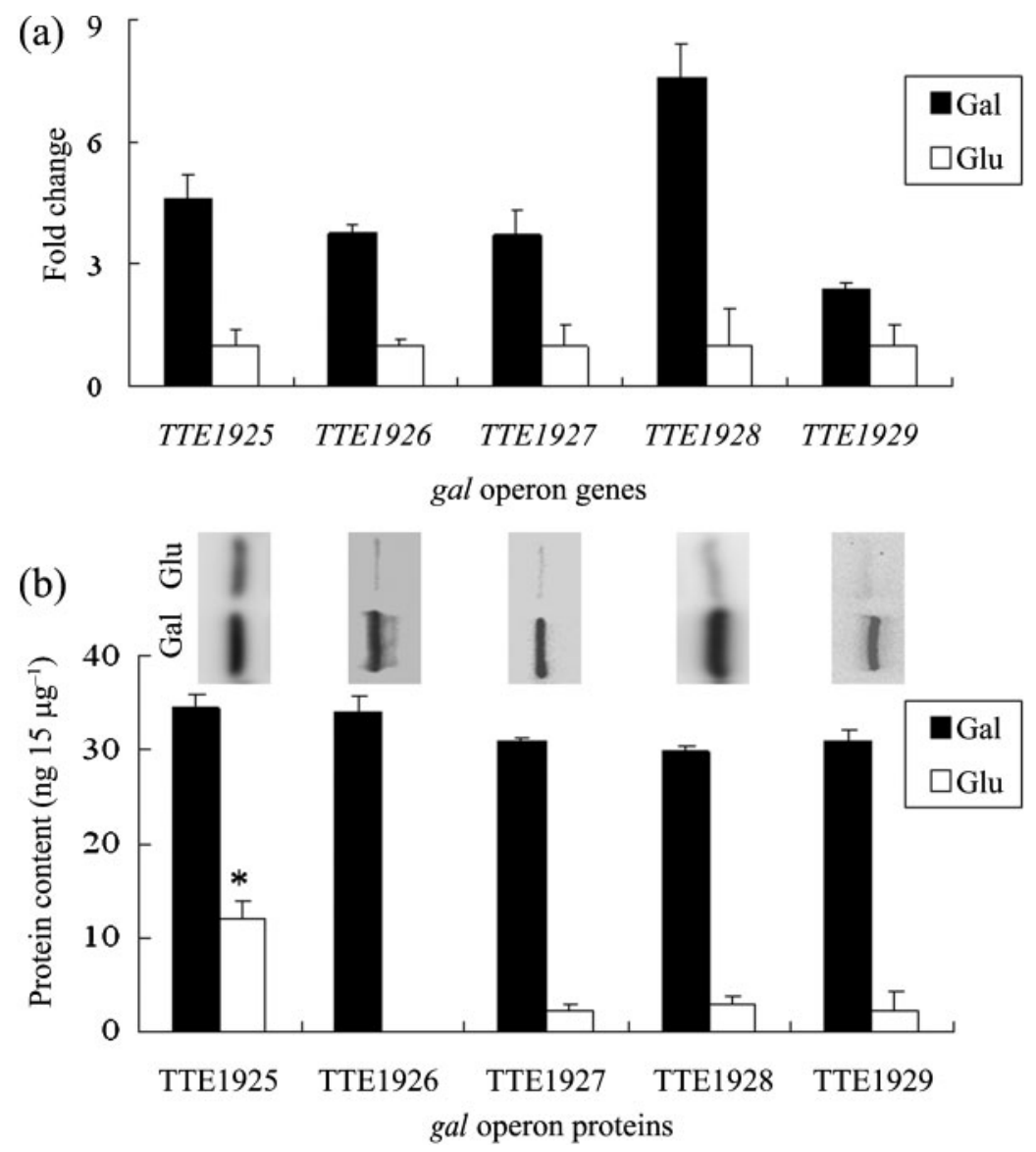

Fig. 2. Gene expression at the mRNA and protein levels of the gal operon of $T$. tengcongensis cultured in glucose (Glu) or galactose (Gal) medium. (a) Relative quantitative comparison of the gal genes by real-time PCR. The $y$ axis represents the fold differences of transcription between the two media normalized to transcription in glucose medium. Mean changes for each gene were obtained from nine runs. (b) Relative quantitative comparison of the gal proteins by Western blotting. The quantitative estimation was based on calibration curves using 10,20,30 and $40 \mathrm{ng}$ recombinant protein of each gene in the Western blot. A total of $15 \mu \mathrm{g}$ protein lysate was loaded into each lane. Mean changes for each protein were obtained from three runs. *Significant differences using Student's $t$ test were defined as $P<0.01$. 


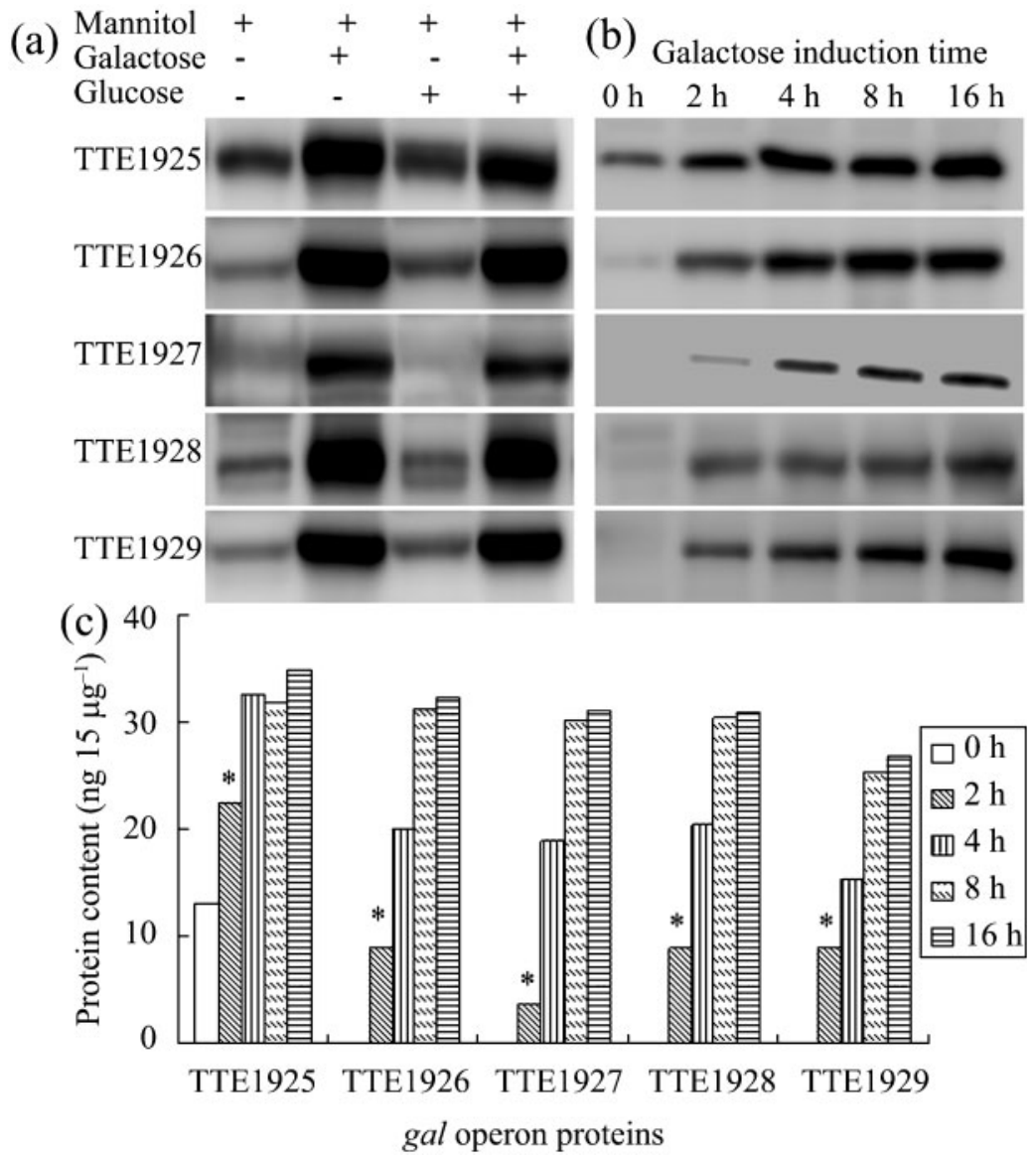

Fig. 3. Western blot analysis of the response of gal proteins to galactose induction. (a) Western blot analysis of $g a /$ proteins in neutral medium with or without galactose or glucose. (b) Dynamic Western blot analysis of gal proteins expressed at the indicated times after galactose induction. (c) Semiquantitative analysis of the intensities of each band recognized by the corresponding antibody. An asterisk indicates a significant difference $(P<0.01)$ between neighbouring times. Mean changes for each protein were obtained from three runs. whereas that of TTE1925 remained relatively stable (data not shown).

\section{Determination of transcriptional start sites in the T. tengcongensis gal operon}

As mentioned above, two promoters have been proposed for the gal operon, $P_{\text {gal }}$ for the whole operon and $P_{\text {gal }}$ for TTE1925 alone. To confirm the prediction, a 5' RACE assay was employed to determine the transcription start sites. The amplified DNA fragments from 5' RACE were examined by $2 \%$ agarose gel and sequenced (data not shown). The sequencing results revealed that right after the specific 5' RACE primer, an adenine, 11 nt upstream of the initiation codon of TTE1929, and a guanine, $20 \mathrm{nt}$ upstream of that of TTE1925, were the transcription starting sites for $P_{\text {gal } 1}$ and $P_{\text {gal }}$, respectively (Fig. 1b).

\section{Functional annotation of the gal proteins in T. tengcongensis}

The GalK activity of recombinant TTE1928 protein was determined over a wide range of temperatures from 30 to $95{ }^{\circ} \mathrm{C}$. As shown in Fig. 4(a), GalK displayed activity from 30 to $95{ }^{\circ} \mathrm{C}$, with a maximum at $80{ }^{\circ} \mathrm{C}$. In addition, the activation energy of GalK was estimated using Arrhenius plots in the temperature range $30-80{ }^{\circ} \mathrm{C}$, and it was found that $44 \mathrm{~kJ} \mathrm{~mol}^{-1}$ was required for the catalytic reaction (Fig. 4a, insert). The kinetic parameters of GalK were estimated. The $K_{\mathrm{m}}$ for galactose was $2.5 \mathrm{mM}$ and for ATP was $5 \mathrm{mM}$. The enzyme activities of GalT and GalE were determined, and were calculated to be 0.087 and 0.130 units (data not shown).

TTE1926 was predicted to be the regulator for the gal operon. EMSA experiments were designed to test whether this protein could interact with the two predicted operators $\left(O_{1}\right.$ and $\left.O_{2}\right)$ and the two control probes $\left(O_{1}{ }^{\prime}\right.$ and $\left.O_{2}{ }^{\prime}\right)$ in vitro. In lanes 2 and 3 of Fig. 5, obvious gel shifts were observed when the two labelled probes were incubated with native protein lysate of $T$. tengcongensis cultured in galactose medium, implying that a protein was bound to these DNA fragments. No gel shift was observed for the $O_{1}{ }^{\prime}$ and $\mathrm{O}_{2}{ }^{\prime}$ controls (data not shown). In addition, the competition experiment was adapted by adding unlabelled probes to the binding mixture. The shifted DNA bands were attenuated due to the addition of unlabelled probe. This result strengthened the argument that the $O_{1}$ and $O_{2}$ fragments indeed have affinity sites for some T. tengcongensis proteins. As determined above, TTE1926 has a DNAbinding motif, an HTH. To prove that the gel shift was caused by an interaction between TTE1926 and the 

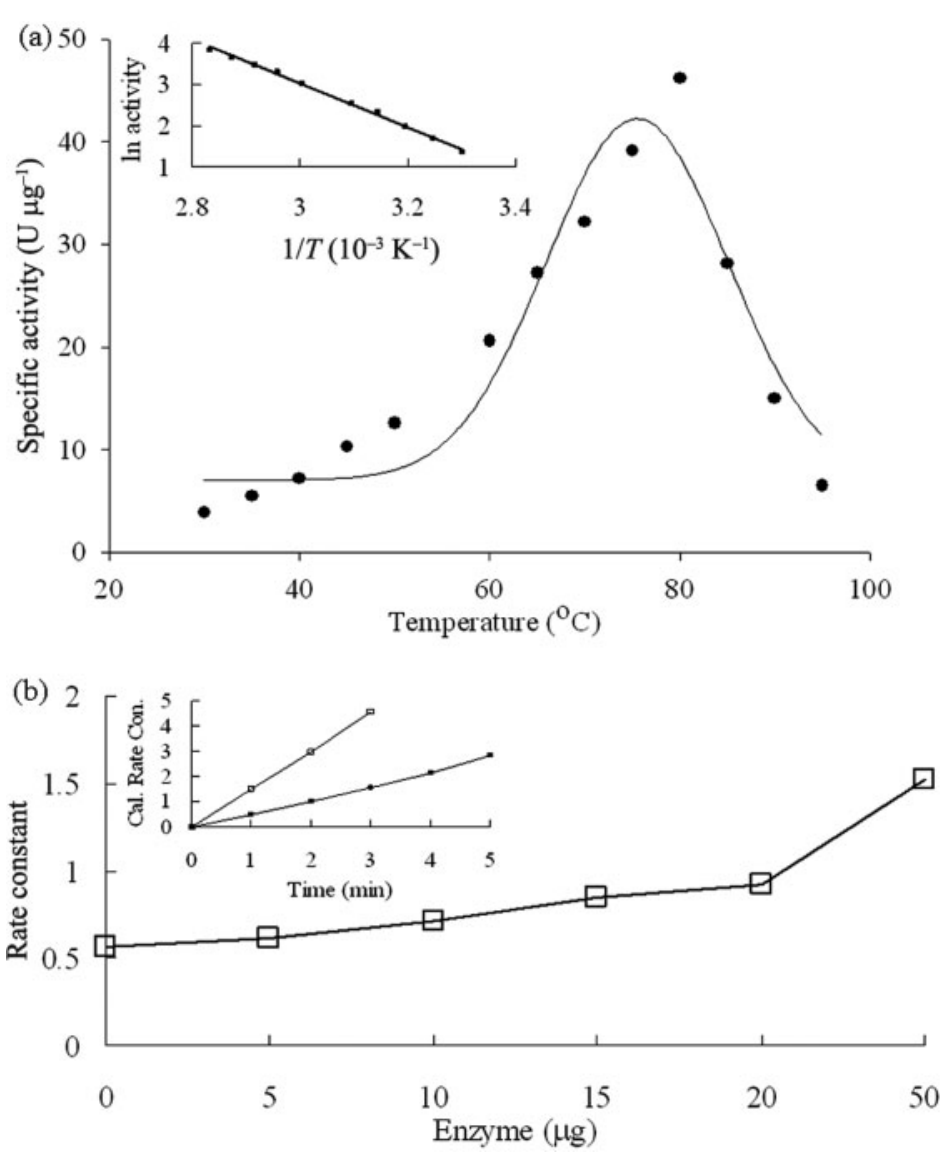

Fig. 4. Enzymic activity assays of recombinant GalK and MR-Tt. (a) Temperature-dependent enzyme activity of recombinant GalK. The insert indicates that the theoretical relationship between enzyme activity and temperature was estimated by Arrhenius plot for GalK. (b) Polarimetric assay of the mutarotase activity of recombinant TTE1925. The insert shows the conversion of $\alpha$ - to $\beta$-glucose with $(\square)$ and without (ם) $50 \mu \mathrm{g}$ recombinant TTE1925. Cal. Rate. Con., calculated rate constant.

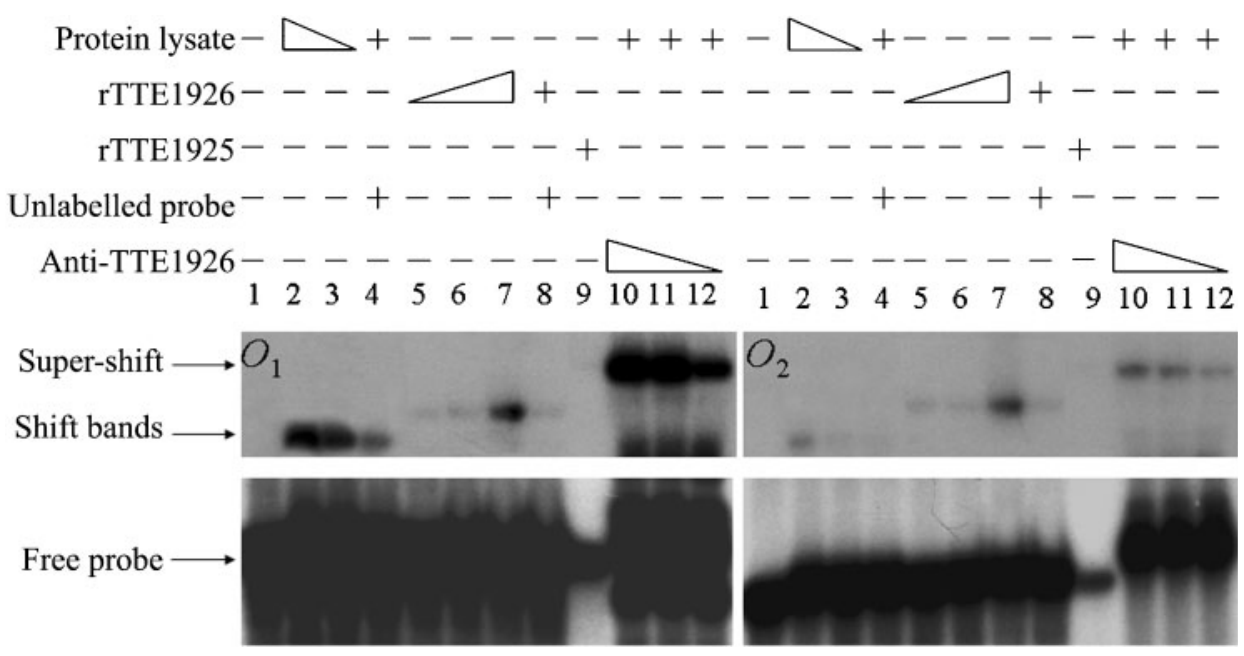

Fig. 5. Interactions between TTE1926 and potential operators, determined by EMSA. Two potential operators were examined, $\mathrm{O}_{1}$ (left) and $\mathrm{O}_{2}$ (right). Upper panels indicate additions of EMSA reaction mixtures. Lane 1 was a blank with probes only. Lanes 2 and 3 were loaded with 50 and $25 \mu \mathrm{g}$, respectively, of protein lysate from galactose medium, while lanes 5, 6 and 7 were loaded with $3.75,37.5$ and $375 \mathrm{ng}$, respectively, of the recombinant TTE1926 protein. Lanes 4 and 8 were competition assays to the mixtures in lanes 2 and 7, respectively, with 10 pmol unlabelled DNA. Lane 9 was loaded with 375 ng recombinant TTE1925 protein as a negative control. Lanes 10,11 and 12 were supershift assays obtained by adding polyclonal antiTTE1926 antibody at three dilutions from stock $(1: 12.5,1: 25$ and $1: 50$, respectively) to the mixture of lane 2 . Upper panels, gel shifts monitored by autoradiography; lower panels, free probes. 
operators, recombinant TTE1926 and TTE1925 proteins were incubated with the labelled probes. As shown in Fig. 5 (lanes 5-9), two bands were shifted in response to the addition of TTE1926, whereas no shifted band was detected for TTE1925 as the negative control. When the antiTTE1926 antibody was added to the binding buffer, the supershifted bands appeared on the gels (Fig. 5, lanes 1012). Taking all the EMSA data together, it was concluded that TTE1926 was likely to interact with the $O_{1}$ and $O_{2}$ fragments to play a regulatory role in the gal operon.

It is well known that $\alpha$-D-galactose is the only substrate for GalK in bacteria. To efficiently utilize galactose in nature, bacteria usually convert $\beta$-D-galactose to $\alpha$-D-galactose. Mutarotase (GalM; EC 5.1.3.3) is a functional enzyme in many bacteria (Brahma \& Bhattacharyya, 2004; Majumdar et al., 2004; Poolman et al., 1990; Thoden \& Holden, 2002), and catalyses the interconversion of the $\alpha$ - and $\beta$-anomers of galactose and glucose. Also, mutarotase is one of the key elements in many gal operons (Bettenbrock \& Alpert, 1998; Thoden \& Holden, 2002; Vaillancourt et al., 2002; Vaughan et al., 2001). Nevertheless, on the basis of the current genomic data for $T$. tengcongensis, there is no mutarotase gene. Is it possible that TTE1925 functions as the mutarotase in T. tengcongensis? A polarimetric assay was carried out. In Fig. 4(b), insert, although spontaneous epimerization of glucose was found without TTE1925, the rotation rates were dramatically stimulated by the addition of TTE1925. The mutarotation rates were concentrationdependent (Fig. 4b). The mutarotation data thus demonstrated that TTE1925 was a novel mutarotase (MR-Tt), even though its protein structure and catalytic mechanism have not yet been fully elucidated.

\section{Protein expression of the gal operon in response to temperature changes}

T. tengcongensis can be cultured over a wide range of temperatures, from 50 to $80{ }^{\circ} \mathrm{C}$, with an optimal temperature at $75{ }^{\circ} \mathrm{C}$ (Xue et al., 2001). A previous investigation that employed a proteomic approach suggested a global change of protein expression in response to temperature elevation (Wang et al., 2007). Expression of the gal proteins in T. tengcongensis cultured in galactose medium at three temperatures, 60,75 and $80{ }^{\circ} \mathrm{C}$, was detected by Western blotting as well as real-time PCR. Except for MR-Tt, the relative mRNA abundance of the gal genes was positively correlated with elevated temperature (Supplementary Fig. S1a). In contrast to the profile at the mRNA level, the relative protein content of these four gal proteins decreased as the temperature rose, as shown in Supplementary Fig. S1(b), and was undetectable at $80{ }^{\circ} \mathrm{C}$. MR-Tt, however, showed a completely different mode, with a relatively higher abundance at $80{ }^{\circ} \mathrm{C}$. These results provoked the question of why the temperature-dependent transcription of the gal genes was so different from the trend in translation. The abundance of a protein results from the balance between its synthesis and degradation. An in vitro protein degradation assay revealed that the recombinant MR-Tt was relatively stable at both 75 and $80{ }^{\circ} \mathrm{C}$, but the other four recombinant gal proteins were quickly degraded at $80{ }^{\circ} \mathrm{C}$ (Supplementary Fig. S1c). This accounts for the low abundance of the four gal proteins at $80{ }^{\circ} \mathrm{C}$.

\section{Proteomic analysis of the DNA-protein complexes of the gal operon}

To identify the operator-binding protein(s), EMSA-LC MS-MS was employed. As shown in Supplementary Table $\mathrm{S} 2$, five proteins were identified in complex $1 \mathrm{~A}$ and three in complex 2A. As expected, GalR was identified in both complex $1 \mathrm{~A}$ and complex $2 \mathrm{~A}$, and this provided additional evidence that GalR was indeed an operator-binding protein. In complex $2 \mathrm{~A}$, a sensory histidine kinase was identified, suggesting that the regulation mechanism for the gal operon was a two-component regulatory system. Some of the other proteins identified from the complexes function in RNA or DNA metabolism, such as the ATPase involved in chromosome partitioning, RNA polymerase subunit alpha, DNA polymerase III subunit alpha and DNA gyrase $\beta$ subunit. These proteins might interact with the probes non-specifically. The ATP : corrinoid adenosyltransferase in complex 1A has not been reported to be related to DNA binding as well as transcription regulation.

\section{DISCUSSION}

In the NCBI database, approximately 50 genomes of thermophilic bacteria are currently available. With a systematic search for genes involved in the Leloir pathway, only seven species containing a relatively complete gal operon or gal-lac regulon were found. To date, only the gallac regulon in $S$. thermophilus has been experimentally examined and the related regulation mechanism extensively investigated (Vaillancourt et al., 2002; Vaughan et al., 2001). No report of a gal operon in thermophilic bacteria is believed to have been published to date. Furthermore, all the gal genes in thermophilic bacteria were analysed by a phylogenetic approach. The phylogenetic trees of GalT, GalK and GalE overlap well, but among the thermophiles GalR is very divergent, indicating that the genes participating in the Leloir pathway are consistent in both mesophiles and thermophiles, although the regulation genes vary in thermophiles (Supplementary Fig. S2). The determination of the unique gal operon structure in T. tengcongensis could, therefore, enhance our understanding of the molecular mechanism of galactose metabolism in thermophiles.

The mesophilic GalRs, identified either in gal operons or in gal-lac regulons, belong to the LacI/GalR regulator family. The $\mathrm{N}$ terminus of LacI-GalR proteins commonly contains the HTH domain, which can specifically bind to certain DNA sequences. A consensus pattern in the $\mathrm{N}$ terminus has been proposed as follows: [LIVM]-x-[DE]-[LIVM]$\mathrm{A}-\mathrm{x}_{(2)}{ }^{-}[\mathrm{STAGV}]-\mathrm{x}-\mathrm{V}-[\mathrm{GSTP}]-\mathrm{x}_{(2)}{ }^{-}[\mathrm{STAG}]-\left[\right.$ LIVMA]- $\mathrm{x}_{(2)^{-}}$ 
[LIVMFYAN]-[LIVMC]. The C-terminal has several regions that could serve for sugar binding as well as for oligomerization. Through sequence alignment and analysis of conserved domains between GalRs of mesophiles and those of $T$. tengcongensis, a sequence comparable to HTH-LacI, ADIAKETN TPPTVTNIV, was found in the N terminus of GalR, with $90 \%$ homology to the consensus sequence. Furthermore, an EMSA experiment was designed to detect the binding of GalR to the putative operators in the gal operon, revealing the interactions of this recombinant protein with the $O_{1}$ and $O_{2}$ fragments. These data are believed to be the first to demonstrate that T. tengcongensis possesses a novel GalR.

GalM could catalyse the interconversion of $\alpha$ - and $\beta$ anomers of galactose and glucose, and has been identified in various species from bacteria to mammals. The X-ray structure of galactose mutarotase from L. lactis at high resolution reveals that the residues $\mathrm{Arg}^{71}$, $\mathrm{His}^{96}$, $\mathrm{His}^{170}$, $\mathrm{Asp}^{243}$ and $\mathrm{Glu}^{304}$ are responsible for anchoring the sugar to GalM. In 34 GalM sequences in SwISS-PROT, a histidine residue is generally located at the centre region and another histidine residue is $\sim 60-80$ aa distant from the centre one at the $\mathrm{N}$ terminus (Thoden \& Holden, 2002). For instance, in E. coli, the mutation of the centre histidine, $\mathrm{His}^{175}$, results in complete loss of enzyme activity, and in L. lactis, mutation of either the $\mathrm{His}^{96}$ or the $\mathrm{His}^{170}$ residue can lead to a significant decrease in enzyme activity (Beebe \& Frey, 1998). Although MR-Tt does not possess any homology to the known GalMs in amino acid sequence, this protein contains a similar pattern of histidine distribution, in which $\mathrm{His}^{129}$ is located at the centre and $\mathrm{His}^{69}$ is 60 aa away from $\mathrm{His}^{129}$. Moreover, the sequence of the recombinant MR-Tt protein exactly followed the predicted ORF (MSVKIEKINFSGWENSIRLS) and then was experimentally proven to have mutarotase activity for $\alpha$-Dglucose. Hence, MR-Tt is a novel mutarotase and a key component of sugar metabolism in T. tengcongensis.

MR-Tt is the last gene in the gal operon and is located upstream of the operon terminator. Expression of this gene was strongly induced by addition of galactose, but was still found at a low level, as either mRNA or protein, in $T$. tengcongensis cultured in glucose medium. A question that arises from this phenomenon is whether MR-Tt is a true member of the gal operon. Experimentally, MR-Tt was cotranscribed with the other operon components, and its gene expression responded sensitively to galactose induction. Therefore, MR-Tt should be a member of the operon. Furthermore, the possibility of an independent promoter for MR-Tt was confirmed by $5^{\prime}$ RACE. Two promoters were deduced in the gal operon, the major one located upstream of the entire operon, $P_{\text {gal }}$, and the minor one upstream of MR-Tt, $P_{\text {gal2 }}$. There is no evidence that dual promoters exist widely in gal operons. For what is believed to be the first time, therefore, it is reported here that dual promoters are indeed located in the T. tengcongensis gal operon.
T. tengcongensis can be cultured from 50 to $80{ }^{\circ} \mathrm{C}$ (Xue et al., 2001), and exhibits temperature-responsive changes in its proteome (Wang et al., 2007). Similar to the proteomic observations, the expression of most gal proteins was decreased significantly with rising temperature. There are manifold impacts upon protein abundance that result from high temperature, such as a short half-life of mRNA (Love et al., 1988), fast degradation of proteins, and inhibition of transcription or translation. By real-time PCR analysis, the mRNA levels of gal genes were found to be upregulated in response to the elevated culture temperatures. Furthermore, an in vitro protein degradation assay showed that gal proteins were significantly more degraded at $80{ }^{\circ} \mathrm{C}$ that at $75{ }^{\circ} \mathrm{C}$, except for MR-Tt. From the above data, it is proposed that the low abundance of the gal proteins except for MR-Tt is likely to be one of the reasons for the slow growth of T. tengcongensis at $80{ }^{\circ} \mathrm{C}$.

\section{ACKNOWLEDGEMENTS}

We are grateful to Zhiguo Cui (College of Chemistry, Peking University) for assistance with the polarimetric experiments. Also, we thank Hua Xiang and Li Guo (Institute of Microbiology, Chinese Academy of Sciences) for expert technical help with EMSA. This work was supported by grants from Key Project of Chinese National Programs for Fundamental Research and Development (2004CB719605) and from National Natural Science Foundation of China (NSFC) (30570399 and 30770467).

\section{REFERENCES}

Ajdic, D. \& Ferretti, J. J. (1998). Transcriptional regulation of the Streptococcus mutans gal operon by the GalR repressor. J Bacteriol 180, $5727-5732$.

Bao, Q., Tian, Y., Li, W., Xu, Z., Xuan, Z., Hu, S., Dong, W., Yang, J., Chen, Y. \& other authors (2002). A complete sequence of the $T$. tengcongensis genome. Genome Res 12, 689-700.

Beebe, J. A. \& Frey, P. A. (1998). Galactose mutarotase: purification, characterization, and investigations of two important histidine residues. Biochemistry 37, 14989-14997.

Bettenbrock, K. \& Alpert, C. A. (1998). The gal genes for the Leloir pathway of Lactobacillus casei 64H. Appl Environ Microbiol 64, 20132019.

Brahma, A. \& Bhattacharyya, D. (2004). UDP-galactose 4-epimerase from Kluyveromyces fragilis. Evidence for independent mutarotation site. Eur J Biochem 271, 58-68.

Frey, P. A. (1996). The Leloir pathway: a mechanistic imperative for three enzymes to change the stereochemical configuration of a single carbon in galactose. FASEB J 10, 461-470.

Lai, K., Willis, A. C. \& Elsas, L. J. (1999). The biochemical role of glutamine 188 in human galactose-1-phosphate uridyltransferase. J Biol Chem 274, 6559-6566.

Li, N., Guo, R., Li, W., Shao, J., Li, S., Zhao, K., Chen, X., Xu, N., Liu, S. \& Lu, Y. (2006). A proteomic investigation into a human gastric cancer cell line BGC823 treated with diallyl trisulfide. Carcinogenesis 27, 1222-1231.

Love, H. D., Jr, Allen-Nash, A., Zhao, Q. A. \& Bannon, G. A. (1988). mRNA stability plays a major role in regulating the temperature- 
specific expression of a Tetrahymena thermophila surface protein. Mol Cell Biol 8, 427-432.

Luesink, E. J., van Herpen, R. E., Grossiord, B. P., Kuipers, O. P. \& de Vos, W. M. (1998). Transcriptional activation of the glycolytic las operon and catabolite repression of the gal operon in Lactococcus lactis are mediated by the catabolite control protein CcpA. Mol Microbiol 30, 789-798.

Mackie, G. \& Wilson, D. B. (1972). Regulation of the gal operon of Escherichia coli by the capR gene. J Biol Chem 247, 2973-2978.

Majumdar, S., Ghatak, J., Mukherji, S., Bhattacharjee, H. \& Bhaduri, A. (2004). UDPgalactose 4-epimerase from Saccharomyces cerevisiae. A bifunctional enzyme with aldose 1-epimerase activity. Eur J Biochem 271, 753-759.

Maxwell, E. S., Kurahashi, K. \& Kalckar, H. M. (1962). Enzymes of the Leloir pathway. Methods Enzymol 5, 16.

Mustapha, A., Hutkins, R. W. \& Zirnstein, G. W. (1995). Cloning and characterization of the galactokinase gene from Streptococcus thermophilus. J Dairy Sci 78, 989-997.

Poolman, B., Royer, T. J., Mainzer, S. E. \& Schmidt, B. F. (1990). Carbohydrate utilization in Streptococcus thermophilus: characterization of the genes for aldose 1-epimerase (mutarotase) and UDPglucose 4-epimerase. J Bacteriol 172, 4037-4047.

Qian, Z., Wang, J. Q., Zhou, C. Q., Ma, Y. H. \& Liu, S. O. (2006). Expression and catalysis of glucokinase of Thermoanaerobacter tengcongensis at different temperatures. Wei Sheng Wu Xue Bao 46, 243-248 (in Chinese).

Rohde, J. R., Trinh, J. \& Sadowski, I. (2000). Multiple signals regulate GAL transcription in yeast. Mol Cell Biol 20, 3880-3886.

Roy, S., Semsey, S., Liu, M., Gussin, G. N. \& Adhya, S. (2004). GalR represses galP1 by inhibiting the rate-determining open complex formation through RNA polymerase contact: a GalR negative control mutant. J Mol Biol 344, 609-618.

Semsey, S., Geanacopoulos, M., Lewis, D. E. \& Adhya, S. (2002). Operator-bound GalR dimers close DNA loops by direct interaction: tetramerization and inducer binding. $E M B O J \mathbf{2 1}$, 4349-4356.

Semsey, S., Tolstorukov, M. Y., Virnik, K., Zhurkin, V. B. \& Adhya, S. (2004). DNA trajectory in the Gal repressosome. Genes Dev 18, 18981907.
Semsey, S., Virnik, K. \& Adhya, S. (2006). Three-stage regulation of the amphibolic gal operon: from repressosome to GalR-free DNA. J Mol Biol 358, 355-363.

Stenger, D., Gruissem, W. \& Baginsky, S. (2004). Mass spectrometric identification of RNA binding proteins from dried EMSA gels. J Proteome Res 3, 662-664.

Thoden, J. B. \& Holden, H. M. (2002). High resolution X-ray structure of galactose mutarotase from Lactococcus lactis. J Biol Chem 277, 20854-20861.

Titgemeyer, F., Reizer, J., Reizer, A. \& Saier, M. H., Jr (1994). Evolutionary relationships between sugar kinases and transcriptional repressors in bacteria. Microbiology 140, 2349-2354.

Vaillancourt, K., Moineau, S., Frenette, M., Lessard, C. \& Vadeboncoeur, C. (2002). Galactose and lactose genes from the galactose-positive bacterium Streptococcus salivarius and the phylogenetically related galactose-negative bacterium Streptococcus thermophilus: organization, sequence, transcription, and activity of the gal gene products. J Bacteriol 184, 785-793.

Vaughan, E. E., van den Bogaard, P. T., Catzeddu, P., Kuipers, O. P. $\&$ de Vos, W. M. (2001). Activation of silent gal genes in the lac-gal regulon of Streptococcus thermophilus. J Bacteriol 183, 1184-1194.

Wang, J., Xue, Y., Feng, X., Li, X., Wang, H., Li, W., Zhao, C., Cheng, X., $\mathrm{Ma}, \mathrm{Y}$. \& other authors (2004). An analysis of the proteomic profile for Thermoanaerobacter tengcongensis under optimal culture conditions. Proteomics 4, 136-150.

Wang, J., Zhao, C., Meng, B., Xie, J., Zhou, C., Chen, X., Zhao, K., Shao, J., Xue, Y. \& other authors (2007). The proteomic alterations of Thermoanaerobacter tengcongensis cultured at different temperatures. Proteomics 7, 1409-1419.

Weickert, M. J. \& Adhya, S. (1993). The galactose regulon of Escherichia coli. Mol Microbiol 10, 245-251.

Wu, K., Lai, X., Guo, X., Hu, J., Xiang, X. \& Huang, L. (2007). Interplay between primase and replication factor $\mathrm{C}$ in the hyperthermophilic archaeon Sulfolobus solfataricus. Mol Microbiol 63, 826-837.

Xue, Y., Xu, Y., Liu, Y., Ma, Y. \& Zhou, P. (2001). Thermoanaerobacter tengcongensis sp. nov., a novel anaerobic, saccharolytic, thermophilic bacterium isolated from a hot spring in Tengcong, China. Int J Syst Evol Microbiol 51, 1335-1341.

Edited by: W. H. Schwarz 